\title{
Philosophiques
}

\section{Philosophie transcendantale et objectivité physique}

\section{Jean Petitot}

Volume 24, numéro 2, automne 1997

URI : https://id.erudit.org/iderudit/027459ar

DOI : https://doi.org/10.7202/027459ar

Aller au sommaire du numéro

Éditeur(s)

Société de philosophie du Québec

ISSN

0316-2923 (imprimé)

1492-1391 (numérique)

Découvrir la revue

Citer cet article

Petitot, J. (1997). Philosophie transcendantale et objectivité physique.

Philosophiques, 24(2), 367-388. https://doi.org/10.7202/027459ar d'utilisation que vous pouvez consulter en ligne.

https://apropos.erudit.org/fr/usagers/politique-dutilisation/ 


\title{
PHILOSOPHIE TRANSCENDANTALE ET OBJECTIVIIÉ PHYSIQUE*
}

\author{
PAR \\ Jean Petitot
}

Dans son remarquable ouvrage Le problème mathématique de l'espace. Une quête de l'intelligible (Springer, 1995) issu de sa thèse de l'EHESS accomplie sous ma direction, Luciano Boi redéploie les problèmes philosophiques liés à l'implication des théories géométriques dans les théories physiques. Il reprend en particulier certains éléments de philosophie transcendantale sur l'actualité desquels j'insiste depuis longtemps. Dans cette note, j'aimerais revenir sur les raisons qui me font penser qu'une lecture transcendantaliste de la physique moderne est possible, non seulement possible mais pertinente, non seulement pertinente mais imposée par la nature même des théories. La physique moderne offre une remarquable leçon de philosophie. Elle commence à rendre l'épistémologie décidable. C'est dire que les thèses philosophiques n'ont plus à être débattues spéculativement mais argumentées à partir de résultats scientifiques.

Disons d'abord un mot sur la possibilité d'opter pour une perspective transcendantaliste alors que c'est une idée reçue que Kant est complètement obsolète depuis les géométries non euclidiennes et la logique de Bolzano. J'adopte une lecture « charitable»des grands philosophes. Ce qui m'intéresse en eux n'est pas tant la lettre de leur texte que l'opérativité de leur pensée relativement à des problèmes fondamentaux. Je les lis comme des savants et j'estime que les enfermer dans la prison exégétique de leur œuvre pour en faire ce que Husserl appelait " des poètes du concept ", c'est les condamner à mort. C'est dire que pour moi Kant est un fondateur et un initiateur, celui de l'orientation transcendantale en philosophie, comme l'étaient pour d'autres orientations Euclide, Descartes, Hume ou Leibniz. On admet bien que les formalistes hilbertiens puissent se réclamer du point de vue axiomatique d'Euclide, ou les phénoménologues husserliens de celui de Descartes, ou encore les empiristes contemporains de celui de Hume sans être pour autant victimes d'une nostalgie archaisante. Et pourtant il est certain que Euclide, Descartes ou Hume sont loin, fort loin, des sciences

Ce texte reprend à la fois mon exposé au colloque organisé par le professeur Robert Nadeau à I'Université McGill en mai 1996 sur l'ouvrage de Luciano Boi et un exposé sur la philosophie de Bernard D'Espagnat à paraitre aux Éditions Diderot. 
modernes. Pourquoi n'en irait-il pas de même pour Kant et le transcendantalisme qui en sont incommensurablement plus proches ? Bref, il n'y a pour moi aucune obstruction de principe à une actualité du transcendantalisme et cela malgré la guerre incessante que lui ont fait les logicistes et positivistes soucieux de rétablir le dogmatisme logique dans ses droits scolastiques pré-critiques.

Ceci dit, il est évident que la possibilité d'une approche transcendantaliste de la physique moderne exige un remaniement profond de Kant et passe en particulier par la disjonction de deux dimensions qui se trouvent chez lui mal distinguées: d'une part la dimension constitutive (doctrine de la constitution des objectivités) et d'autre part la dimension cognitive (le fait que c'est une conscience qui est constituante).

Kant a découvert la problématique de la constitution, à savoir qu'il existe des principes prescriptifs (et non pas descriptifs) de la réalité physique. Comme l'affirmait Hans Reichenbach, il s'agit là d'un « résultat philosophique éminent ". Mais, chez Kant, le constitutif reste fondé sur une base cognitive (théorie représentationaliste, doctrine des facultés, etc.). Comme le soulignait Schlick, chez Kant les principes constitutifs sont caractéristiques de notre conscience représentationnelle. D'où le subjectivisme transcendantal.

Dans ma lecture de Kant je mets au premier plan la problématique de la constitution et au second plan celui des structures (innées ou non) de la conscience constituante. Certes l'espace et le temps comme formes des phénomènes sont " mentaux "au sens où notre appareil perceptif est un appareil de mesure du signal optique (et même un extraordinaire appareil de mesure). Mais leur fonction est très particulière. Elle est de formater les informations (ce que Kant appelait le divers pur de la sensation). À ce titre, ce sont des formes complètement " désubjectivisées ${ }^{1}$ . Il en va de même en Mécanique quantique où ce n'est pas parce que les phénomènes n'existent que mesurés par des appareils que la réalité physique se réduit pour autant à un a solipsisme d'appareil.

L'approche constitutive me paraît done être exemplairement juste pour la mécanique classique et parfaitement prolongeable à la physique moderne, à condition bien sûr de la recentrer sur le problème des conditions de possibilité de l'expérience et de la connaissance scientifique, c'est-à-dire sur celui de l'auto-limitation de la connaissance objective relativement à toute ontologie et, par conséquent, sur son incompatibilité avec une logique naturelle de choses en soi substantielles, individuées et indépendantes possédant des propriétés.

1. D'ailleurs les travaux de neurosciences montrent à quel point la représentation neuronale de l'espace est compliquée et n'a rien à voir avec la géométrie. Elle repose sans doute sụr des phénomènes de synchronisation d'oscillateurs neuraux. Voir Petitot [1994a]. 


\section{Les moments transcendantaux de la physique théorique classique}

Pour voir qu'une approche transcendantale de la physique moderne est possible, revenons un instant sur le grand texte physique de Kant, à savoir les Premiers Principes Métaphysiques de la Science de la Nature (PPM) si profondément analysés par Jules Vuillemin dans Physique et Métaphysique kantiennes ${ }^{2}$. Essayons d'en formuler brièvement le sens en un langage un peu plus moderne.

\section{Phoronomie (Cinématique)}

Dans la cinématique, qui spécifie les catégories de la quantité et les a Axiomes de l'intuition o réglant la fonction des grandeurs extensives, deux problèmes sont traités.

(a) La façon dont la mesure advient, sous forme d'arithmétisation, de coordonnées, et surtout de métrique, à l'espace et au temps purement phénoménologiques. L'espace comme forme de présentation et medium de manifestation (intuition pure) devient géométrie (intuition formelle) en vue de la physique et sa structure euclidienne est inséparable des principes de la mécanique (principe d'inertie et géodésiques rectilignes).

(b) Les mouvements rectilignes uniformes et le groupe d'invariance de la relativité galiléenne. Cela recouvre d'une part les symétries de l'espace/temps : translations temporelles, translations et rotations spatiales, etc. (Il faut insister sur le fait que Kant est le premier philosophe à avoir affirmé, contre les dogmatismes logiques métaphysiques (leibniziens par exemple), que l'existence de symétries spatiales était constitutive de l'objectivité physique). Cela recouvre d'autre part le groupe proprement cinématique des transformations galiléennes (mouvements rectilignes uniformes). D'où l'affirmation du rôle constitutif du principe de relativité. Ainsi que le note J. Vuillemin : a c'est le principe de la phoronomie qui fournit la véritable démonstration de l'Esthétique transcendantale » et " c'est la relativité du mouvement qui rend transcendantalement nécessaire la subjectivité de l'espace [son idéalité transcendantale] ${ }^{3}$ m.

On voit à quel point on est loin de tout idéalisme subjectif et solipsiste.

Notons d'ailleurs que, pour Kant, la loi d'addition des vitesses dans la relativité galiléenne n'avait rien d'évident et constituait même un problème central. En effet, les vitesses sont des grandeurs intensives et non pas extensives. Leur additivité (leur vectorialité) doit donc être démontrée en accord avec leur intensivité et cela ne va pas de soi ${ }^{4}$. Le mouvement n'est pas

2 Vuillemin [1955]

3. Vuillemin [1955], p. 59-60.

4. On dirait maintenant que les vitesses appartiennent, non pas à $\mathrm{R}^{3}$, mais à ses espaces vectoriels tangents $T_{x} \mathbf{R}^{3}$ (qui ne sont isomorphes à $\mathbf{R}^{9}$ que parce que ce dernier est lui-même un espace vectoriel). 
un mode de l'espace et l'additivité n'est pas que géométrique. Elle est cinématique.

\section{Dynamique}

\section{Le débat avec Leibniz}

Comme qualité (et non plus comme quantité), la matière est remplissement de l'espace. Ce remplissement est très différent d'une simple a occupation "(anti-cartésianisme). C'est un processus dynamique et énergétique propre à a l'intériorité " substantielle de la matière. C'est ici l'un des points où le débat de Kant avec Leibniz est le plus serré. Pour Leibniz, l'espace est imaginaire. L'intériorité substantielle, la substance-force réelle, est hor's espace, bien qu'elle s'exprime spatialement. Kant maintient cette intériorité substantielle, mais seulement à titre de fondement. Toutefois, comme elle est d'ordre nouménal, elle ne peut pas être introduite dans le domaine de l'objectivité. Elle doit être déterminée à travers sa seule extériorisation (sa spatio-temporalisation à travers le mouvement). Il s'agit par conséquent de conquérir un concept purement spatiotemporel de Dynamique qui ne relève plus de la chose en soi. Mais cela implique la fondation de la Dynamique dans la Phoronomie. Or, comme y insiste J. Vuillemin : « que la Dynamique présuppose la Phoronomie, cela signifie la possibilité d'une révolution copernicienne concernant la catégorie de substance, révolution qui est sans doute le cour de l'idéalisme kantien " (p. 87).

C'est effectivement là que les catégories de la qualité se disjoignent irréversiblement du concept traditionnel (ontologique et métaphysique) de substance. Le remplissement de l'espace/temps par la matière est une tension dynamique pour l'occupation. Il est le résultat du conflit de forces fondamentales attractives et répulsives engendrant la cohésion des corps, leurs phases matérielles et leurs interactions. Ces forces fondamentales a primitives internes - - qui doivent être bien distinguées des forces a dérivatives externes "que sont les forces mécaniques - ont un être opaque qui s'exprime phénoménologiquement par des qualités, en l'occurrence par des grandeurs intensives.

Kant va donc élaborer dans la Dynamique le traitement mathématique des grandeurs intensives. Mais l'on voit que cela laisse entièrement ouvert le problème du concept dynamique de matière. L'intériorité substantielle génératrice des qualités dynamiques demeure en fait hors construction. C'est un problème central abordé dans l'Opus Postumum ${ }^{5}$ et légué par Kant à l'avenir.

5. J'ai analysé ailleurs cette * Critique de la Raison Physique * qu'est l'Opus Postumum. Voir Petitot [1990b] et [1991c]. 


\section{L'Opus Postumum et la physique des formations phénoménales}

Dans ces réflexions ultimes, Kant envisage entre autres une genèse physique de la phénoménalité elle-même, phénoménalité conçue comme la manifestation d'une intériorité substantielle de la matière pour un dispositif de perception. L'OP est hanté par un retour de l'en soi dynamique leibnizien éliminé dans la $C R P$ et les $P P M$, mais par un retour prenant l'allure d'une conquête en quelque sorte physique de cet en soi.

Dans la théorie du phénomène du phénomène, il s'agit non plus tant de légalisation objective des phénomènes que de leur origine, de leur genèse à partir d'un fondement. Ce fondement considéré jusque là comme d'ordre nouménal doit se convertir en fondement physique. Avec lui, les conditions de possibilité de l'expérience traitées dans la CRP doivent devenir les conditions de possibilité de la phénoménalité même manifestant les objets physiques de la mécanique. Comme l'affirme le père François Marty dans sa Préface à sa traduction de l' $O P$, elles doivent permettre de " comprendre ce qu'est un réel manifesté " (p. 378).

Pour ce faire, Kant a envisagé d'appliquer l'Analytique transcendantale non plus, comme dans les $P P M$, à l'objet régional qu'est le mouvement mais au nouvel objet régional qu'est le système des forces fondamentales conçues comme forces primitives motrices internes à la matière.

Ces forces primitives intérieurement motrices sont beaucoup plus fondamentales que les forces extérieurement motrices et mécaniques traitées dans les PPM. Elles sont dynamiques: " toutes les forces primitives de la matière sont dynamiques: " les forces mécaniques sont seulement dérivées "(p. 37). Elles ne sont pas motrices à travers des déplacements. Elles sont: "motrices en leurs parties, les unes par rapport aux autres dans l'espace occupé par la matière "(p. 25).

Elles sont génératrices de perception et expliquent les Anticipations de la perception (p. 38). Par extériorisation, elles engendrent des forces mécaniquement motrices tombant, elles, sous la législation des Analogies de l'expérience et des Postulats de la pensée empirique (p. 38). Grâce à elles on peut comprendre la présence (c'est-à-dire la stabilité), la formation et la composition des corps car : " elles forment des corps qui déterminent eux. mêmes leur espace, selon la quantité et la qualité » (p. 39).

On peut comprendre: “ une matière déterminant elle-même par ses propres forces sa figure et sa structure, et résistant à leur changement, originellement et de manière uniforme " (p. 47) (résolution du problème initial de la Dynamique dans les $P P M$.

Le fondement physique engendre les formes de l'intuition elles-mêmes puisqu'il rend l'espace perceptible (p. 61). L'Esthétique transcendantale se trouve convertie en objet physique issu d'une causalité physique plus profonde. La matière n'est plus seulement, comme dans les $P P M$, le substrat 
du mouvement: a Elle est ce qui fait de l'espace un objet des sens empiriquement intuitionnable, le substrat de toute intuition empirique externe avec conscience $\times$ (p.110).

\section{Dynamique et géométrie différentielle}

Revenons aux PPM. Dans la Mécanique, la matière se trouve réduite à la masse. Cela permet la construction mathématique du mouvement tout en faisant l'économie de celle, dynamique, de la substantialité ${ }^{6}$.

Les catégories de la qualité et les principes associés que sont les « Anticipations de la Perception * se spécifient ici par le principe que l'être physique s'externalise à travers des grandeurs intensives comme la vitesse ou l'accélération. Le lien organique, systématique, avec la cinématique s'exprime alors comme un principe de covariance relativement au groupe d'invariance de l'espace-temps. Ainsi apparaît, après celle du principe de relativité, l'interprétation transcendantale d'un autre principe fondamental, à savoir que l'être physique doit être décrit par des données différentielles variant de façon covariante. Bref, la Dynamique nous explique que, pour des raisons transcendantales, la Mécanique doit être une géométrie différentielle (et non pas une logique des propriétés).

\section{Mécanique}

La Mécanique, qui spécifie les catégories de la relation et les principes associés que sont les "Analogies de l'expérience ", explique la genèse des objets physiques proprement dits. Elle repose, nous venons de le voir, sur la réduction de la matière à la masse. La masse devient " sujet ultime dans l'espace ", le mouvement devient son a prédicat déterminant ", et, dans la mesure où ce prédicat est spatio-temporel, la matière se trouve bien traitée scientifiquement à partir de sa seule phénoménalité. La matière n'est plus une matière physique "seconde "animée par en-dessous par une materia prima extrinsèquement spatiale. Inertiale, elle devient en quelque sorte une unité matière-espace-temps.

Cela permet de construire mathématiquement les catégories « dynamiques * (c'est-à-dire non « mathématiques »), catégories qui ne pouvaient être que schématisées au niveau de la $C R P$.

6. Dans sa remarquable étude Inertia, the communication of Motion, and Kant's third law of mecharics, Howard Duncan a analysé en détail la façon dont opère dans les $P P M$ le fait que le concept de matière, comme tension dynamique pour l'occupation de l'espace, ne soit pas dynamiquement constructible. Une véritable ontologie de la matière exigerait que l'on puisse ramener ultimement les corps matériels à un effet de forces dynamiques fondamentales opérant sur une sorte de a fluide • énergétique primordial (d'où l'anti-atomisme de Kant). Cela étant selon Kant mathématiquement impossible, la Dynamique évince le problème et change de niveau pour se restreindre au niveau purement mécanique du mouvement (des trajectoires). Ce faisant, la genèse physique de la matière est remplacée par sa description scalaire comme masse inertiale. Cela permet de construire mathématiquement le mouvement et la communication du mouvement sans construire pour autant le concept de matière. C'est cette dernière construction qui fait l'objet des réflexions visionnaires de l' $O P$. 
Ce point est essentiel. Les catégories mathématiques concernent les intuitions pures et donc l'essence comme a premier principe interne de tout ce qui appartient à la possibilité d'une chose .

Les catégories dynamiques concernent au contraire la nature comme a premier principe interne de tout ce qui appartient à l'existence d'une chose $\mathrm{s}$.

Dans la $C R P$, à la différence des catégories mathématiques qui concernent l'essence, les catégories dynamiques posent l'existence, la conditionnent tout en la laissant indéterminée. Cela implique qu'elles ne soient pas constructibles. Elles ne s'appliquent qu'à l'objet en général et ne sont que schématisables. Mais elles deviennent constructibles lorsqu'elles s'appliquent a à une détermination supplémentaire ", en l'occurrence le mouvement, "contenant une intuition pure ".

La construction commence par une réinterprétation complète de la catégorie de substance? ${ }^{?}$ À travers son schématisme temporel qui en fait un principe de permanence, celle-ci s'identifie désormais aux principes de conservation des grandeurs physiques, c'est-à-dire aux principes physiques d'invariance.

On ne saurait trop insister sur l'importance épistémologique de cette réinterprétation. Elle rompt avec toutes les approches logico-ontologiques qui considèrent que la science est une prédication sur les états de choses d'une réalité indépendante. Ici, le concept de substance devient la source des lois de conservation, lois qui, une fois traduites en équations, épuisent l'essentiel du contenu théorique des théories physiques.

Quant au principe de causalité, il se trouve retraduit par le principe d'inertie et la loi de Newton.

Enfin, quant au principe de communauté, il se trouve retraduit d'abord par le principe de l'égalité de l'action et de la réaction puis par le principe de l'interaction universelle qui spécifie le schème de la simultanéité.

La simultanéité pose d'ailleurs pour Kant un problème difficile. En effet, on doit en garantir l'objectivité. Or, pour cela, il faut coordonner les temps locaux en un temps cosmologique global. L'espace et le temps sont donnés comme infinis au niveau de l'Esthétique transcendantale. Mais en tant qu'espace et temps de l'objectivité physique, ils sont, si on les considère globalement, de nature cosmologique. Or l'univers ( le monde ") n'est pas un concept objectif pour Kant (voir les antinomies cosmologiques de la Dialectique transcendantale). Ce n'est qu'une Idée. (Cela a même conduit Kant à renier ses considérations cosmologiques pré-critiques que tous les spécialistes s'accordent pourtant à juger visionnaires.)

7. Il ne faut pas oublier que, pour Kant, le concept métaphysique traditionnel (aristotélicoscolastique) de substance comme support d'accidents n'était qu'une simple hypotypose symbolique (voir Critique de la Faculté de Juger \$59). 


\section{Phénoménologie}

Ce que les PPM développent sous le titre de Phénoménologie concerne les catégories de la modalité (possible, réel, nécessaire) et les principes associés que sont les a Postulats de la pensée empirique en général ".

1. Ce qui s'accorde avec les conditions formelles de l'expérience (intuitions et catégories) est possible.

2. Ce qui est en cohésion avec les conditions matérielles de l'expérience (la sensation) est réel.

3. Ce dont la cohésion avec le réel est déterminé suivant les conditions générales de l'expérience est nécessaire (existe nécessairement). (On notera le retour de l'existence tout à la fin du système des principes.)

Insistons sur le fait que le concept de réalité est ici une catégorie modale qui ne possède de sens objectif que relativisé à une stratégie constitutive.

Cinématiquement parlant, à cause de la relativité, le mouvement n'est pas un prédicat réel mais seulement possible. Il ne peut pas être interprété comme une transformation réelle de l'état interne réel du système et de certaines de ses propriétés qui seraient des propriétés mécaniques intrinsèques. En réduisant la matière à la masse, en mettant entre parenthèses les « forces primitives internes "et en ne traitant que des a forces dérivatives externes ", la Mécanique s'interdit de pouvoir ramener les mesures sur le mouvement à une détermination de l'état interne du système. C'est pourquoi on peut à la fois affirmer le mouvement et le nier sans aucune contradiction logique. Autrement dit, la relativité du mouvement rend inacceptable l'interprétation spontanée (ontologisante) d'énoncés comme a le corps $S$ " a " telle position ou telle vitesse ", où " avoir " est pris au sens de " posséder une propriété . De tels énoncés ne supportent pas la contreefactualité puisque leur valeur de vérité présuppose que l'on ait choisi un repère inertial, c'est-à-dire que l'on ait précisé les conditions des mesures. Il existe par conséquent pour Kant un conflit irréductible entre l'objectivité physique et la logique naturelle (prédicative) du sens commun. Position (spatiale ou temporelle) absolue ainsi que vitesse absolue (d'un mouvement rectiligne uniforme) ne sont pas des observables. On peut certes considérer qu'il reste correct, d'un point de vue pratique, de parler « comme si " la localisation et le mouvement étaient des propriétés. Mais ce "réalisme empirique " de l'espace ne peut en aucun cas se doubler d'un " réalisme transcendantal ». La fameuse thèse de al'idéalité transcendantale " de l'espace ne fait qu'exprimer philosophiquement cette conséquence (à l'époque traumatisante) du principe de relativité.

La dynamique fournit en revanche des critères de réalité du mouvement, car les forces sont réelles. Cette réalité est gouvernée par des lois mécaniques qui sont nécessaires, la nécessité n'étant pas ici une modalité logique mais une modalité transcendantale, une nécessité conditionnelle, relative à la contingence radicale de l'expérience. 
On voit à quel point la proscription du transcendantal par l'empirisme logique a pu être dommageable pour la philosophie de la physique. Alors que Kant avait fait un effort vertigineux pour clarifier les problèmes fondamentaux posés par le statut très particulier de l'objectivité physique (disjonction objectivité/ontologie, caractère prescriptif de la légalisation catégoriale, rôle constitutif des symétries, des contraintes de covariance et des lois de conservation, caractère modal des mesures et de la réalité physiques, inadaptation de la logique eu égard à sa nature " dogmatique ", etc.), au lieu de poursuivre son effort parallèlement aux progrès des sciences on a (et cela malgré l'importance de travaux comme ceux de Cassirer sur la Relativité) remis le compteur à zéro et réimposé un logicisme reflétant une ontologie incompatible avec l'objectivité physique. Tout l'effort du criticisme a donc dû être recommencé. Étant donné l'irrationalisme engagé des philosophies “ continentales », il n'a pu l'être que par les physiciens euxmêmes qui ont entièrement réinventé le sens authentique des moments transcendantaux de l'expérience et des éléments critiques de la connaissance. Ceux-ci ont effectivement “ donné une leçon "à la philosophie, dans tous les sens du terme.

Le “tabou "anti-transcendantal rend parfois la situation épistémologique un peu confuse. Par exemple, Baas Van Fraassen a redécouvert le caractère fondamentalement modal des concepts physiques d'observable et de réalité. Il faut faire la différence entre les énoncés d'attribution d'état et reux d'attribution de valeur d'observable : " states can be identified in terms of observables, but cannot be identified with them ${ }^{8}$.

Les transitions du possible à l'actuel lors des mesures sont décrites par les états mais ne sont pas des transitions d'état. De même qu'en mécanique classique les résultats des mesures sur le mouvement ne peuvent pas être interprétés ontologiquement comme des prédicats réels des corps, de même en Mécanique quantique les résultats de mesure ne sont pas interprétables ontologiquement comme des prédicats réels des états. Normalement de telles thèses devraient être considérées comme d'esprit transcendantaliste. Mais étant donné le « tabou ", on doit les présenter comme une nouvelle forme d'empirisme. 


\section{Les Principes de symétrie et la construction de catégories «dynamiques" de substance, de causalité et d'interaction}

\section{La téléologie transcendantale des théories physiques}

Mon hypothèse de travail est qu'il existe également une structure transcendantale des théories physiques postérieures à la Mécanique rationnelle et même, plus précisément, qu'il existe une histoire transcendantale de ces théories. Celle-ci possède, me semble-t-il, trois aspects :

(i) l'approfondissement des moments transcendantaux ;

(ii) la remise en cause de l'exclusion d'un dynamisme interne et la théorisation du système des forces fondamentales primitives internes à la matière (et non pas dérivatives externes comme les forces mécaniques);

(iii) la construction au sens fort des catégories dynamiques de substance, de causalité et d'interaction, c'est-à-dire leur engendrement à partir d'un élargissement de l'interprétation mathématique des catégories mathématiques, c'est-à-dire en définitive d'un enrichissement des principes de relativité.

La conséquence en est une involution de la structure transcendantale des théories dans leurs constructions mathématiques. Cette structure transcendantale n'est plus lisible que mathématiquement. Si l'on met entre parenthèses le contenu mathématique spécifique des théories physiques, on n'a donc plus aucune possibilité d'accéder à leur signification transcendantale.

J'ai étudié ailleurs trois exemples particulièrement spectaculaires.

\section{Le théor ème de Noether et la construction de la catégorie de substance}

Le premier exemple d'approfondissement de moment transcendantal est fourni par le théorème de Noether. Ce théorème fondamental possède selon moi un sens non seulement physique, mais également transcendantal éminent. Il montre en effet que, sous la double condition :

(i) de pouvoir décrire mathématiquement la relation entre Cinématique et Dynamique par l'action d'un groupe de relativité sur l'espace de phases du système physique considéré ;

(ii) de disposer d'une formulation variationnelle (lagrangienne ou hamiltonienne) de la Mécanique (c'est-à-dire des lois du mouvement), alors il existe une corrélation explicite entre les symétries et les grandeurs conservées. Cela correspond à la construction (au sens fort) de la catégorie de substance, déjà réduite par Kant aux lois de conservation.

De façon plus précise, le formalisme lagrangien-hamiltonien qui domine la physique permet d'engendrer les grandeurs physiques d'un système à partir de ses grandeurs cinématiques extensives (positions) et intensives (vitesses). Il permet en quelque sorte de convertir du "mathématique" (de l'essence) en " dynamique " (en existence). Le théorème de Noether dit que si 
un lagrangien est invariant sous un groupe de relativité à un paramètre (c'està-dire de dimension 1), alors il existe une grandeur physique conservée au cours du mouvement (une intégrale première). Il relie donc :

(i) principes de relativité (inobservabilité de grandeurs cinématiques absolues) ;

(ii) symétries (invariance du lagrangien);

(iii) lois de conservation (observabilité) de grandeurs physiques corrélatives.

C'est en quelque sorte $l e$ théorème transcendantal qui donne raison à Kant au-delà de tout ce qu'il pouvait imaginer et espérer.

Les exemples les plus classiques corrèlent:

(i) la conservation de l'énergie au groupe d'invariance des translations temporelles ;

(ii) la conservation de l'impulsion au groupe d'invariance des translations spatiales ;

(iii) la conservation du moment angulaire au groupe d'invariance des rotations spatiales.

J'ai exposé ailleurs ${ }^{9}$ les aspects modernes de ce théorème en géométrie symplectique et en particulier le formalisme de l'application moment, c'est-àdire la façon dont on peut associer à un groupe de Lie qui opère symplectiquement sur un espace de phases des intégrales premières a priori et cela de façon indépendante de tout hamiltonien (mais si un hamiltonien est invariant sous l'action du groupe alors il possède ces intégrales premières). Ces résultats renforcent considérablement le contenu synthétique a priori du théorème de Noether.

\section{La relativité générale et la construction de la catégorie de cause (du concept de force)}

Mon deuxième exemple est celui de la Relativité Générale (RG). On a souvent dit que la $\mathrm{RG}$ avait rendu impossible une lecture transcendantale de la physique moderne. Je pense toutefois que c'est exactement le contraire qui est vrai. La structure transcendantale de la $\mathrm{RG}$ est manifeste et remarquable. Mais elle change profondément le contenu kantien des moments transcendantaux. Comme l'a bien vu Cassirer dans son ouvrage de 1921, Zur Einsteinschen Relativitätstheorie, elle les approfondit. Ce n'est que si l'on interprète ces contenus de façon fixiste dans le cadre d'une interprétation cognitive innéiste que l'on peut conclure à l'obligation d'abandonner une lecture transcendantale. En fait, transcendantalement parlant, la RG correspond à la construction de la catégorie de cause (de force). 
Dans l'interprétation transcendantale, cette construction consiste à ramener la force à un principe de relativité, c'est-à-dire à une généralisation de l'Esthétique transcendantale. C'est bien ce que fait la RG. En RG, les moments transcendantaux que sont les Axiomes de l'intuition (avec la Cinématique correspondante) et les Anticipations de la Perception (avec la Dynamique correspondante) sont passés du niveau global et métrique, qui était le leur en mécanique newtonienne, au niveau local et différentiable sousjacent. Cela n'était évidemment possible qu'après la conquête mathématique de ce niveau par Riemann. Le groupe de relativité de la théorie devient alors le groupe des difféomorphismes de l'espace-temps. Les contraintes de covariance deviennent par conséquent beaucoup plus importantes et cela permet de ramener la force, donc la catégorie de cause, à un principe d'inertie généralisé. Les a priori géométriques ne sont plus dès lors de nature métrique mais de nature différentielle et concernent, par exemple, la cohomologie des formes différentielles ${ }^{10}$.

De façon un peu plus précise, soit $\mathbf{E}$ l'espace-temps muni de sa métrique localement minkowskienne $g_{\mu v}$. Si $e_{\alpha}$ est une base de l'espace vectoriel tangent à $\mathrm{E}$ en $x T_{x} \mathrm{E}$ et si $\omega^{\alpha}$ est la base duale de l'espace cotangent $T^{*} \mathrm{E}$, le tenseur de courbure de Riemann est défini en termes de composantes par $R^{\alpha} \beta \gamma \delta=<\omega^{\alpha},[\nabla \gamma, \nabla \delta] e \beta>$ (où $\nabla$ est la dérivation covariante, où $[\bullet, \bullet]$ est à la fois le crochet de Lie de l'algèbre de Lie des champs de vecteurs et le commutateur des opérateurs différentiels et où, pour $\omega \in T^{*}{ }_{x} \mathrm{E}$ et $v \in T_{x} \mathrm{E}$, $<\omega, \nu>=\omega(\nu))$. Pour $\omega \in T^{*}{ }_{x} \mathrm{E}$ et $u, v, w \in T_{x} \mathrm{E}$, on a $R(\omega, u, v, w)=<\omega$, $\mathbf{R}(v, w) u>\operatorname{avec} R(v, w)=\left[\nabla_{v}, \nabla_{w}\right]-\nabla[v, w]$.

Par contraction du tenseur de courbure de Riemann, on construit le tenseur de courbure de Ricci $R_{\mu v}=R_{\mu}^{\alpha} \alpha v$. Par une seconde contraction, on construit la courbure scalaire $R=R_{\mu}{ }^{\mu}$. Le tenseur de courbure d'Einstein est donné par $G=$ Ricci $-1 / 2 g R(g=|\operatorname{det}(g \mu v)|)$. Il satisfait pour des raisons $a$ prioriques - purement géométriques, en fait cohomologiques - , les identités de Bianchi $\nabla G \equiv 0$.

Dans les équations d'Einstein $G=8 \pi T$ (où $T$ est le tenseur d'impulsion énergie), l'identité $\nabla T \equiv 0$ formule le principe de la conservation de l'énergie (l'expression de la catégorie de substance). Elle devient une conséquence de la géométrie de l'espace-temps, car elle dérive de l'a priori synthétique $\nabla G \equiv 0$.

Comme Hilbert l'a montré dès 1921 et comme cela a été développé dans les années 1960 par Wheeler, Arnowitt, Deser et Misner" ${ }^{11}$ il est possible de dériver la métrique $g_{\mu \nu}$ de l'espace temps $E$ d'un principe variationnel en prenant la courbure scalaire $R$ comme densité lagrangienne. L'action est alors :

10. Dans Petitot [1992a], j’ai analysé en détail, dans cette perspective, la géométro-dynamique de Wheeler et son débat avec Grünbaum (pour ce dernier, voir aussi plus bas, section V).

11. Voir Misner, Thorne, Wheeler [1973]. 


$$
S=\frac{1}{16 \pi} \int_{\mathrm{E}} R \sqrt{|g|} d^{4} x .
$$

On déduit de cette formule un système hamiltonien (de dimension infinie) sur l'espace fonctionnel des métriques.

Cela montre qu'en $R G$ la métrique ne relève plus, comme cela était le cas en mécanique rationnelle, des catégories mathématiques, mais bien des catégories dynamiques. Elle devient une entité physique qui doit elle-même être déterminée. Mais cela ne signifie pas pour autant l'invalidation de la logique transcendantale. Bien au contraire. Cela signifie seulement que l'Esthétique transcendantale est passée du niveau métrique global au niveau différentiable local. Comme nous l'avons dit plus haut, le groupe de relativité n'est plus désormais le groupe de Galilée ou le groupe de Lorentz mais le groupe $\operatorname{Diff}(\mathrm{E})$ des difféomorphismes de $\mathrm{E}$. C'est donc à ce niveau que se situe désormais le synthétique a priori. Ce déplacement de niveau permet d'absorber la catégorie de force dans un principe de relativité élargi et, par là même, de la construire.

\section{Les théories de jauge et la construction de la catégorie d'interaction}

L'exemple est encore plus spectaculaire avec les théories de jauge où c'est non seulement la catégorie de force (comme dans la relativité générale) mais aussi la catégorie d'interaction qui se trouvent ramenées à des principes de symétries élargis. Comme l'explique Yuri Manin ${ }^{2}$ : “ From a philosophical point of view, one can speak of a new wave of geometrization of physical thought which for the first time is sweeping far beyond the boundaries of general relativity ${ }^{\prime}$.

Depuis les travaux pionniers de Chen Ning Yang et Robert Mills (1954) sur l'invariance de jauge concernant l'isospin, il existe dans les théories de jauge deux classes de champs :

1) les champs fermioniques de matière qui sont interprétés comme des sections de fibrés sur l'espace-temps. Les coordonnées des fibres sont les degrés internes de liberté. Le groupe structural (c'est-à-dire le groupe de symétrie des fibres) exprime les symétries internes des particules.

2) les champs bosoniques de jauge qui sont des champs d'interactions véhiculées par des particules virtuelles d'échange (des bosons) et sont interprétés comme des connexions sur ces fibrés. Les particules véhiculant les interactions sont par conséquent les quanta des champs-connexions sur les fibrés de matière.

Le lagrangien de Yang-Mills est la norme de la courbure des connexions. Il est invariant sous l'action du groupe de jauge et l'espace-temps y contribue

12 Manin [1988]. 
comme champ de jauge à travers la courbure scalaire de sa connexion. Les dérivations covariantes permettent d'exprimer géométriquement les interactions.

Plus précisément, rappelons qu'en théorie quantique des champs, on dispose d'une chaîne de procédures de déterminations objectives conduisant de principes constitutifs à des modèles explicites.

Les principes de relativité et de symétrie fournissent des Lagrangiens $L$, ou mieux des densités de lagrangien $\mathrm{L}\left(\varphi, \partial_{\mu} \varphi\right)$ dépendant des champs $\varphi(x, t)$ considérés et de leurs dérivées premières $\partial_{\mu} \varphi$. Cela permet de définir des actions $S(\Gamma)$ sur des chemins $\Gamma$ conduisant d'un état initial $\varphi \dot{ }=\varphi\left(x, t_{1}\right)$ à un état final $\varphi f=\varphi\left(x, t_{2}\right)$ :

$$
S(\Gamma)=\int \mathrm{L} \quad d^{4} x=\int_{t_{1}}^{t_{2}} \int_{\mathrm{R}^{3}} \mathrm{~L}\left(\varphi, \partial_{\mu} \varphi\right) d^{3} x d t .
$$

Les axiomes de la mécanique quantique conduisent alors de l'action $S(\Gamma)$ à la formule de Feynman (intégrale de chemin) pour l'amplitude de probabilité de transition ( $h$ est la constante de Planck):

$$
\left\langle\varphi_{\Gamma} \mid \varphi_{i}\right\rangle=\int_{\Gamma} \exp \left(\frac{2 i \pi}{h} S(\Gamma)\right) d \Gamma
$$

Il s'agit d'une intégrale fonctionnelle dans l'espace fonctionnel des chemins. Elle n'est pas bien définie comme objet mathématique (c'est l'un des principaux problèmes de la théorie quantique des champs), mais elle fournit néanmoins un algorithme de calcul extraordinairement puissant.

Il est bien connu que cette formule (qui est analogue aux fonctions de partition $Z$ de la mécanique statistique) encode une quantité énorme d'information. Il est possible d'en dériver un nombre considérable de modèles explicites, quantitatifs et prédictifs des phénomènes en utilisant des outils mathématiques appropriés comme par exemple :

(i) les développements perturbatifs ;

(ii) le théorème de Wick disant que tous les moments d'une loi de probabilité gaussienne peuvent s'exprimer en fonction de ses moments d'ordre 2 ;

(iii) le théorème de la phase stationnaire disant qu'une intégrale oscillante $\mathrm{e}^{\mathrm{i} \tau(x)}$ se concentre pour $\tau \rightarrow \infty$ sur les points critiques de la phase $\varphi(x)$;

(iv) le groupe de renormalisation.

On rencontre ici un splendide exemple d'une détermination objective conduisant de principes constitutifs à des modèles spécifiques et diversifiés : les principes constitutifs (groupes de relativité, symétries) 
fournissent des lagrangiens, qui fournissent à leur tour des intégrales de Feynman, qui fournissent elles-mêmes les modèles :

$A$ priori constitutifs $\rightarrow$ Groupes de relativité et symétries $\rightarrow$ Lagrangiens $\rightarrow$ Action $\rightarrow$ Intégrales de chemins $\rightarrow$ Modèles spécifiques de phénomènes ${ }^{13}$.

Dans ce contexte, les théories de jauge ont réussi à construire a priori les interactions en faisant dépendre les symétries internes des systèmes (qui sont des symétries globales apparemment non spatiotemporelles associées aux nombres quantiques des particules) de la position spatiotemporelle. Si on localise ainsi ces symétries internes et si l'on exige que les théories demeurent invariantes, on doit introduire des termes correctifs. On constate alors que ceux-ci redonnent exactement les termes d'interaction. Les forces et les interactions apparaissent ainsi de façon générale comme dérivables de principes de conservation locaux ${ }^{14}$.

Le cas le plus simple (découvert par Hermann Weyl) est celui du " couplage minimal " entre un électron et un champ électromagnétique $F$. Soit $\psi$ la fonction d'onde de l'électron. Son évolution est régie par l'équation de Dirac. Le lagrangien de Dirac $\mathrm{L}_{D}$ est invariant sous la symétrie interne globale $\psi \rightarrow e^{-i e \vartheta} \psi$ (où le $e$ en exposant est la charge de l'électron et $\vartheta$ une phase). Le groupe des symétries internes est le groupe des phases, c'est-àdire le groupe commutatif $\mathrm{U}(1)$ des rotations du cercle. D'autre part, le lagrangien de Maxwell (champ électro-magnétique) $\mathrm{L}_{M}$ est invariant sous une transformation de jauge $A \rightarrow A+d \Lambda$, où $A$ est le potentiel vecteur du champ électro-magnétique $F, \Lambda$ une fonction sur $\mathrm{E}$ et $d \Lambda$ la différentielle de $\Lambda$. Si l'on admet que le facteur de phase $\vartheta$ peut dépendre de la position spatiotemporelle $x \in \mathrm{E}$, alors le Lagrangien de Dirac $\mathrm{L}_{D} n$ 'est plus invariant. Mais le terme de correction qui apparaît peut être exactement compensé par la transformation de jauge $A \rightarrow A+d \vartheta$. Il s'agit là d'une sorte de " miracle * dont la signification tant physico-mathématique que transcendantale est remarquable.

Géométriquement, le potentiel vecteur $A$ s'interprète comme une connexion définie sur un fibré vectoriel $\mathrm{F}$ au-dessus de l'espace temps $\mathrm{E}$ et le champ $F$ s'identifie à la courbure de cette connexion. Le groupe de relativité est maintenant encore plus large que le groupe Diff $(\mathrm{E})$. C'est le groupe - dit groupe de jauge - des automorphismes du fibré $F$ de base E. Cet élargissement permet de ramener la catégorie dynamique d'interaction à un principe de relativité, et donc de la construire. Dans le cas non abélien, le groupe des symétries internes $G(G=\mathrm{SU}(2), \mathrm{SU}(3)$, etc.), n'est plus commutatif. Cela introduit des difficultés profondes dans la théorie, mais les idées principales subsistent.

13. Voir par exemple Zuber Itzykson [1985] et Le Bellac [1988].

14. Voir par exemple Quigg [1983]. 
Le cas est encore plus spectaculaire avec la théorie des supercordes ${ }^{15}$. En fait, toute symétrie supplémentaire enrichit de façon considérable le contenu mathématique des théories physiques. Par exemple, en théorie des supercordes, la simple reparamétrisation des cordes conduit à introduire la théorie des surfaces de Riemann et, plus particulièrement, la théorie de Teichmüller des espaces de modules qui classifient les différentes structures complexes compatibles à une même structure différentiable. Il en va de même avec l'interprétation donnée récemment (1994) par Edward Witten de la symétrie électrique $\leftrightarrow$ magnétique de Montonen-Olive (1977). Elle conduit à l'introduction de formes modulaires et a déjà eu des conséquences considérables pour la compréhension de la structure des variétés différentiables de dimension $4^{16}$.

Ce sont ces stratégies remarquables de détermination objective des phénomènes qui font dire aux physiciens, par exemple à Michio Kaku ${ }^{17}$ :

the secret of this mystery /celui des théories unifies/ most likely lies in the power of gauge symmetry.

Nature demands symmetry.

Symmetry, instead of being a purely aesthetic feature of a particular model, now becomes its most important feature (p. 8).

Bref, au moyen des formalismes variationnels, du théorème de Noether, des intégrales de Feynman et des théories de jauge, il a été possible de construire une véritable ontogenèse formelle de la réalité physique. Une telle construction convertit le synthétique a priori en règles d'engendrement de modèles explicites diversifiés. Les contraintes mathématiques sont si fortes (renormalisabilité, élimination des anomalies, mécanisme de Higgs et ruptures spontanées de symétries conférant une masse aux bosons de jauge, etc.) qu'il est souvent possible d'inférer le bon choix du groupe de symétrie de la théorie à partir d'un tout petit nombre de données empiriques significatives.

\section{Interpretation transcendantale du conventionalisme géométrique de Poincaré}

Cette possibilité de construction (au sens fort) des catégories dynamiques en physique théorique a de nombreuses conséquences épistémologiques. Je n'en évoquerai ici qu'une seule, concernant l'interprétation transcendantale du conventionalisme. Je rappelle les deux thèses d'Henri Poincaré sur la physique mathématique exposées, entre autres, dans les chapitres IV et V de La Science et l'Hypothèse : « L'espace et la géométrie ", "L'expérience et la géométrie : :

1. La thèse selon laquelle la géométrie appliquée à la physique est conventionnelle (ni vraie ni fausse), que l'on peut décrire les mêmes contenus

\footnotetext{
15. Voir par exemple Kaku [1988] et Love Bailin [1994], ainsi que Petitot [1992b]

16. Voir Witten [1994] et Donaldson [1996]

17. Kaku [1988.
} 
factuels physiques dans des cadres géométriques alternatifs. En tant que convention, une géométrie fixe un langage de description et ne possède pas de vérité empirique expérimentale ;

2. La thèse que le concept de groupe est une forme a priori de l'entendement.

C'est surtout dans la conclusion de “ L'espace et la géométrie * qu'est exposée la thèse du conventionalisme géométrique :

Ce qui est l'objet de la géométrie, c'est l'étude d'un * groupe • particulier ; mais le concept général de groupe préexiste dans notre esprit au moins en puissance. Il s'impose à nous, non comme forme de notre sensibilité, mais comme forme de notre entendement.

Seulement, parmi tous les groupes possibles, il faut choisir celui qui sera pour ainsi dire

l'étalon auquel nous rapporterons les phénomènes naturels.

L'expérience nous guide dans ce choix qu'elle ne nous impose pas; elle nous fait reconnaitre non quelle est la géométrie la plus vraie, mais quelle est la plus commode.

Cette thèse est approfondie dans “ L'expérience et la géométrie " où Poincaré explique que les principes de la géométrie ne sont pas des faits expérimentaux. On peut toujours exprimer un même fait physique en changeant la convention du cadre géométrique et en changeant les lois physiques (par exemple on peut garder la géométrie euclidienne et ne pas garder le principe que les rayons lumineux sont des géodésiques).

Comme Luciano Boi l'a bien montré, ce point de vue avait déjà été admirablement anticipé par Clifford à la fin du siècle dernier : il existe une équivalence entre :

(i) des causes physiques de changements dans un espace posé a priori comme plat ;

(ii) une géométrie non triviale (courbe) de l'espace.

Les expériences physiques portent toujours sur des corps et jamais sur l'espace. Elles ne peuvent donc pas décider de la géométrie.

Bref, les thèses sont :

1. la géométrie est une convention, c'est-à-dire un a priori (grammatical si on veut) de l'expérience. Elle fixe un langage de description et ne possède pas de vérité expérimentale. L'a priori n'a ici rien à voir avec un a priori logique ou un a priori innéiste cognitif. C'est un a priori au sens de conditions de possibilité déterminantes ;

2. l'a priori de la géométrie se ramène essentiellement à l 'a priori des groupes : groupes d'invariance, groupes de relativité, groupes de symétrie des théories physiques ;

3. l'a priori n'étant pas inné et ne pouvant pas être décidé par l'expérience, il doit être choisi ;

4. Le critère du choix est pragmatique : c'est celui de la commodité.

Mais nous avons vu que, en ce qui concerne l'évolution de la physique mathématique, on peut observer, à travers le mouvement toujours plus accentué et profond de géométrisation de la physique, une évolution qui 
motive le choix des conventions géométriques et possède le statut philosophique d'une réduction à l'a priori mathématique des contenus physiques empiriques.

Par exemple, dans le cas de la relativité générale, la conventionalité de la géométrie pour la physique signifie que l'on choisit une métrique de façon à ce que les rayons lumineux soient des courbes de longueur nulle et les mouvements en chute libre des géodésiques. Comme l'a expliqué Adolph Grünbaum dans sa critique de la Géométrodynamique de John Archibald Wheeler, Charles Misner et Kip Thorne ${ }^{18}$, ces deux classes de trajectoires sont empiriquement données et ce n'est que le choix conventionnel de la métrique qui les qualifie géométriquement. Certes. Mais la qualification einsteinienne est pourtant plus motivée que d'autres, car elle ramène les mouvements gravitationnels à des mouvements inertiaux. En fait la RG est la théorie de jauge du groupe de Poincaré.

La dépendance de l'objectivité physique par rapport à des conventions fixant la qualification géométrique des données empiriques, en particulier le fait que les symétries des théories sont mathématiquement déterminantes pour le contenu physique de ces théories, est, répétons-le, la forme moderne de l'idéalité transcendantale de l'espace. Cette idéalité transcendantale s'oppose aussi bien à un réalisme ontologique qu'à un idéalisme subjectif (psychologique). Elle est prescriptive, c'est-à-dire à la fois conventionnelle et normative (déterminante).

On peut donc aller plus loin dans l'interprétation du conventionalisme géométrique que ne l'a fait Poincaré en adoptant un point de vue pragmatique de “ commodité ». Nous avons vu en effet qu'il existe un télos de la géométrisation en physique : transformer des principes de symétrie en principes dynamiques.

Paraphrasant des affirmations de Jean-Marie Souriau à propos de la quantification géométrique, on peut dire que : " philosophiquement [la géométrisation] c'est ramener la physique à des symétries géométriques pour faire de la physique a priori (c'est-à-dire " rationnelle ") ".

Autrement dit, comme l'affirme encore Souriau : il n'y a rien de plus dans les théories physiques que les groupes de symétrie si ce n'est la construction mathématique qui permet précisément de montrer qu'il n'y a rien de plus ${ }^{19}$ ".

Cela est une parfaite définition de la réduction à l'a priori : il n'y a rien de plus si ce n'est les mathématiques permettant de montrer qu'il n'y a rien de plus. Ce principe est devenu le principe de découverte majeur des théories physiques contemporaines. S'il y a des structures physiques empiriques supplémentaires, c'est qu'il y a des symétries supplémentaires et que l'on n'a pas pris un groupe de symétrie approprié.

18. Grünbaum [1973], chapitre XXII : General Relativity, Geometrodynamics and Ontology.

19. Pour la quantification géométrique, voir Souriau [1975] et Weinstein [1977]. 
Le rôle déterminant des symétries en physique confère à l'objectivité physique un statut très particulier, qui oppose cette objectivité à toute ontologie substantialiste d'étants singuliers et individués, existant de façon transcendante comme entités séparées. Cette vieille tradition métaphysique aristotélicienne est incompatible avec la physique moderne. L'objectivité physique est transcendantale au sens où c'est une objectivité a faible "qui inclut dans son concept d'objet les conditions d'accès et les conditions de possibilité de détermination de ses objets. Plus précisément: ce qui est accessible à la théorie, son contenu positif, y est défini négativement, c'est-àdire par ce qui lui est inaccessible (à cause des symétries). Les symétries imposent une auto-limitation à ce que la théorie peut connaître et dire qu'elles sont constitutives, c'est dire que ce que la théorie peut connaitre est déterminé par ce que la théorie ne peut pas connaître. Il s'agit là du principe de base qui disjoint l'objectivité physique de toute ontologie. On peut le qualifier de principe galoisien dans la mesure où un principe analogue a été formulé pour la première fois de façon claire par Galois dans la façon dont celui-ci a complètement repensé le problème de la résolution des équations algébriques.

Cette nature galoisienne a été excellemment soulignée par l'éminent. spécialiste de géométrie symplectique et des travaux de Witten qu'est Daniel Bennequin, en particulier dans son long article en hommage à Thom : "Questions de physique galoisienne ". Dire philosophiquement que l'objectivité physique est transcendantale, c'est dire techniquement qu'elle est galoisienne.

Pour moi, le sens moderne du synthétique a priori concerne cette caractéristique des théories physiques modernes, à savoir que le maximum d'économie et de puissance théorique s'obtient en ramenant les contenus physiques d'abord à des principes dynamiques (conservations, forces, interactions) et ensuite ces principes eux-mêmes à des conséquences physiques de symétries qui expriment que l'on doit pouvoir éliminer dans les théories physiques les éléments mathématiques conventionnels (coordonnées, repères, jauges, etc.) nécessaires à la description. Le fait que, par exemple dans une théorie de jauge à la Yang-Mills, une propriété physique comme une self-interaction de bosons de jauge soit reliée au fait que le groupe des symétries globales internes est non cornmutatif, est, pour moi, un exemple typique de synthétique a priori. Cela n'a rien à voir avec une nécessité logique. D'ailleurs déjà chez Kant, le synthétique a priori était toujours corrélatif de la contingence radicale de l'expérience.

École des Hautes Études en Sciences Sociales 


\section{BIBLIOGRAPHIE}

ABRAHAM, R., MARSDEN, J. 1978, Foundations of Mechanics, Benjamin Cummings, New.York, Reading.

ALLISON, H.E., 1983. Kant's Transcendental Idealism. An Interpretation and Defense, New-Haven, Yale University Press.

ARNOLD, V., 1976. Méthodes mathématiques de la mécanique classique, Moscou, Mir.

BAILIN, D., LOVE, A., 1994. Supersymmetric Gauge Field Theory and String Theory, Institute of Physics Publishing, London.

BENNEQUIN, D., 1994. "Questions de physique galoisienne " Passion des Formes, à René Thom (M. Porte ed.), 311-410, E.N.S. Éditions FontenaySaint Cloud.

BITBOL, M., 1996. Mécanique quantique. Une introduction philosophique, Paris, Flammarion.

BOHR, N., 1935. Physical Review, 48, 696.

BOI, L., 1995. Le problème mathématique de l'espace, Berlin, Springer.

BRITTAN, G., 1978. Kant's Theory of Science, Princeton University Press.

CASSIRER, E., 1918. Kants Leben und Lehre, Kant's Life and Thought (trad.J. Haden), Yale University Press, 1981.

COHEN-TANNOUDJI, G., SPIRO, M., 1986. La Matière - Espace - Temps, Paris, Fayard.

DONALDSON, S. K., 1996. « The Seiberg-Witten equations and 4-manifold topology ", Bulletin of the American Mathematical Society, 33, 1, 45-70.

DUNCAN, H., 1984. a Inertia, the communication of Motion, and Kant's third law of mechanics ", Philosophy of Science, 51, 93-119.

d'ESPAGNAT, B., 1985. Une incertaine réalité, Paris, Gauthier-Villars.

d'ESPAGNAT, B., 1994. Le réel voilé, Paris, Fayard.

FOLSE, H.J., 1978. "Kantian Aspects of Complementarity ", Kant-Studien, 69, 58-66.

GOMEZ, R.J., 1986. a Beltrami's Kantian View of Non-Euclidean Geometry ", Kant-Studien, 77, 1, 102-107.

GRÜNBAUM, A., 1973. Philosophical Problems of Space and Time, Dordrecht - Boston, Reidel.

HONNER, J. 1982. a The Transcendental Philosophy of Niels Bohr », Studies in History and Philosophy of Science, 13, 1, 1-29.

ICMP, 1995. XI International Congress of Mathematical Physics (D. Iagolnitzer, ed.), International Press.

ITZYKSON, C., ZUBER, J.B., 1985. Quantum Field Theory, Singapour, Mc Graw-Hill.

JAMMER, M., 1974. The Philosophy of Quantum Mechanics, John Wiley and Sons.

KAKU, M., 1988. Introduction to Superstrings, New-York, Springer.

KANT, E, 1980-1986. Euvres philosophiques (F. Alquié ed.), Paris, Bibliothèque de la Pléiade, Gallimard.

KANT, I., 1781-1787. Kritik der reinen Vernunft, Kants gesammelte Schriften, Band III, Preussische Akademie der Wissenschaften, Berlin, Georg Reimer, 1911.

KANT, I., 1786. Metaphysische Anfangsgründe der Naturwissenschaft, Kants gesammelte Schriften, Band IV, Preussische Akademie der Wissenschaften, Berlin, Georg Reimer, 1911. Premiers Pincipes métaphysiques de la Science de la Nature, Trad. J. Gibelin, Paris, Vrin, 1971. 
KANT, I., 1790. Kritik der Urtheilskraft, Kants gesammelte Schriften, Band V, Preussische Akademie der Wissenschaften, Berlin, Georg Reimer, 1913. Critique de la Faculté de Juger, trad. A. Philonenko, Paris, Vrin, 1979.

KANT, I., 1796-1803. Opus Postumum, trad. F. Marty, Paris, Presses Universitaires de France, 1986.

LE BELLAC, M., 1988. Des phénomènes critiques aux champs de jauge, Paris, InterÉditions - C.N.R.S.

LOCHAK, G., 1994. La géométrisation de la physique, Paris, Flammarion.

MANIN, Y., 1988. Gauge Field Theory and Complex Geometry, Springer.

MARSDEN, J., 1974. Applications of Global Analysis in Mathematical Physics, Berkeley, Publish or Perish.

MISNER, C.W., THORNE, K.S., WHEELER, J.A., 1973. Gravitation, San Francisco, Freeman.

OMNES, R., 1994. Philosophie de la science contemporaine, Paris, Gallimard.

PETITOT, J., 1990a. « Le Physique, le Morphologique, le Symbolique. Remarques sur la vision ", SC [1990], 139-183.

PETITOT, J., 1990b. "Logique transcendantale, Synthétique a priori et Herméneutique mathématique des Objectivités n, Fundamenta Scientiae, (numéro en l'honneur de L. Geymonat), 10, 1, 57-84.

PETITOT, J., 1991a. “Idéalités mathématiques et Réalité objective. Approche transcendantale ", Hommage à Jean-Toussaint Desanti, (G. Granel ed.), 213-282, Éditions TER, Mauvezin.

PETITOT, J., 1991b. La Philosophie transcendantale et le problème de l'Objectivité, Entretiens du Centre Sèvres, (F. Marty ed.), Paris, Éditions Osiris.

PETITOT, J., 1992a. Physique du Sens, Éditions du CNRS, Paris.

PETITOT, J., 1992b. a Actuality of Transcendental Aesthetics for Modern Physics ", 1830-1930:A Century of Geometry, (L. Boi, D. Flament, J.-M. Salanskis eds), Berlin, New-York, Springer.

PETITOT, J., 1992c. “ Continu et Objectivité. La bimodalité objective du continu et le platonisme transcendantal „, Le Labyrinthe du Continu, (J.-M. Salanskis, H. Sinaceur eds.), 239-263, Springer, Paris.

PETITOT, J., 1994a. "La sémiophysique : de la physique qualitative aux sciences cognitives ", Passion des Formes, à René Thom (M. Porte éd.), 499545, E.N.S. Éditions Fontenay-Saint Cloud.

PETITOT, J., 1994b. a Esthétique transcendantale et physique mathématique ${ }^{\prime}$ Neukantianismus. Perspektiven und Probleme (E.W. Orth, H. Holzhey Hrsg.), 187-213, Königshausen \& Neumann, Würzburg.

PETITOT, J., 1995. "Pour un platonisme transcendantal ", L'objectivité mathématique. Platonisme et structures formelles, (M. Panza, J-M. Salanskis eds.), 147-178, Paris, Masson.

PHILONENKO, A., 1972. L'œuvre de Kant, Paris, Vrin.

POINCARE, H., 1902. La Science et l'Hypothèse, Paris, Flammarion.

QUIGG, C., 1983. Gauge Theories of the Strong, Weak, and Electromagnetic Interactions, Reading, Benjamin-Cummings.

SOURIAU, J.M., 1975. Géométrie symplectique et physique mathématique, Coll. Internat. du C.N.R.S., 237, Paris.

VAN FRAASSEN, B. C., 1991. Quantum Mechanics. An Empirist View, Clarendon Press, Oxford.

VUILLEMIN, J., 1955. Physique et Métaphysique kantiennes, Paris, Presses Universitaires de France.

WEINSTEIN, A., 1977. Lectures on Symplectic Manifolds, C.B.M.S., Conf. Series, Am. Math. Soc., 29, Providence. 
WEIZSÄCKER, C.F. von, 1979. Die Einheit der Natur, Munich, Hauser.

WEYL, H., 1922. Space - Time - Matter, New-York, Dover.

WIREDU, J.E., 1970. « Kant's Synthetic a priori in geometry and the rise of non-euclidean geometries ", Kant-Studien, 61, 1, 5-27.

WITTEN, E., 1994, a Electric-Magnetic Duality in Four Dimensional Gauge Theories », ICMP 1994, 155-162. 